\title{
Hematogenous pasteurella haemolytica brain abscess
}

\author{
Mehdi Darmoul, Atef Ben Nsir, Mohamed Kilani, Mohamed Nejib Hattab \\ Department of Neurosurgery, CHU Fattouma Bourguiba Monastir, Tunisia
}

\begin{abstract}
Pasteurella infections are common in domestic animals and very rare in human. We report a hematogenously acquired Pasteurella haemolytica brain abscess, mimicking brain tumor on magnetic resonance imaging, in an 18-year-old female patient known with cardiac interventricular communication, without recent history of animal contact. The outcome was good after abscess complete removal and antimicrobials therapy for 6 weeks. To the best of our knowledge, this is the first reported case of $P$. haemolytica brain abscess.
\end{abstract}

Key words: Brain abscess, hematogenous route, Pasteurella haemolytica

\section{Introduction}

Brain abscess due to Pasteurella is very rare. About 11 cases of Pasteurella multocida brain abscess, caused frequently by penetrating trauma or contiguous infection, were reported in the literature.

Herein, we describe the first case of hematogenously acquired brain abscess caused by Pasteurella haemolytica in an 18-year-old woman.

\section{Case Report}

The present case report is about an 18-year-old female patient, from urban area, with past history of cardiac interventricular communication (IVC), was admitted to our department for two episodes of generalized tonic-clonic seizures which occurred 1 month before admission for the first and the day of admission for the second.

There are no signs of intracranial hypertension, fever or neurological deficit and no recent history of sinusitis, otitis, dental infection or animal contact.

\begin{tabular}{|l|l|}
\hline \multicolumn{2}{|c|}{ Access this article online } \\
\hline Quick Response Code: & Website: \\
\hline & www.ruralneuropractice.com \\
\cline { 2 - 2 } & \\
\hline
\end{tabular}

The seizures were brought under control after oral treatment with sodium valproate.

On admission, the patient's temperature was $36.8^{\circ} \mathrm{C}$ and her blood pressure was $110 / 60 \mathrm{mmHg}$. Neurological and general examinations were unremarkable and no skin lesions were noted.

The chest X-ray and electrocardiogram were normal. The echocardiogram showed a small IVC without signs of cardiac failure or endocarditis.

Blood analysis revealed white blood cell count $8300 / \mu 1$ with predominance of neutrophils, hemoglobin $12.7 \mathrm{~g} / \mathrm{dl}$ and C-reactive protein $1 \mathrm{mg} / \mathrm{l}$.

Brain magnetic resonance imaging showed a right parietal lesion hypointense on T1 sequence, enhanced on its periphery by contrast with moderate surrounding edema [Figure 1].

Pre-operative suggested first diagnosis was glial tumor and the patient underwent right parietal craniotomy. After dura opening the lesion puncture found a hard consistency and did not aspirate any liquid, then a complete removal of a well-limited mass was performed. The mass examination found a thick wall containing yellow-green liquid, redressing the diagnosis for brain abscess.

The post-operative course was uneventful and the patient was initially treated intravenously with cefotaxime, fosfomycin and metronidazole.

Address for correspondence: Prof. Mehdi Darmoul, Department of Neurosurgery, CHU Fattouma Bourguiba, Rue 1er Juin, Monastir 5000, Tunisia. E-mail: mehdi.darmoul@laposte.net 
Aerobic and anaerobic culture of abscess fluid yielded a pure growth of $P$. haemolytica [Figure 2] sensitive to all antimicrobials to which it was tested including cefotaxime and fosfomycin. This therapy was continued intravenously for 1 month and relayed orally by ciprofloxacin and rifadine for 2 weeks. Control computed tomography scan confirms complete removal of the abscess.

\section{Discussion}

Pasteurella is a facultative anaerobic Gram-negative coccobacillus which is best known as part of oral cavity and gastrointestinal flora of many domestic animals such as cats, dogs..., etc. ${ }^{[1]}$

It is a common cause of infections in animals and rarely causes infections in humans. ${ }^{[1-3]}$

The first Pasteurella species was isolated in 1878 by Pasteur in fowl cholera. ${ }^{[1,2]}$ Brugnatelli in his study have reported the first human case of Pasteurella infection causing puerperal sepsis in 1913. ${ }^{[1,2]}$

P. multocida is the most frequent human pathogen. Most infections arise from animal bites or scratches and remain localized. ${ }^{[1-4]}$

Serious deep-seated infections occur occasionally including respiratory tract infections, liver abscess, peritonitis, urinary tract infections, septicemia..., etc. ${ }^{[4]}$ Central nervous system infections occur infrequently; representing less than $5 \%$ of non-bite-related cases and are mainly meningitis. ${ }^{[4]}$ Brain abscesses are very rare. ${ }^{[4,5]}$

There are only about 11 published case reports of brain abscess caused all by P. multocida. ${ }^{[5]}$

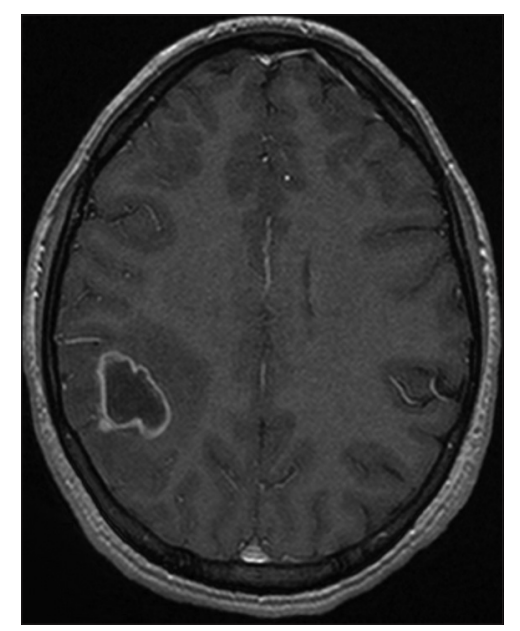

Figure 1: Axial T1-weighted magnetic resonance imaging with gadolinium showing a right parietal rim-enhancing lesion
P. haemolytica also called Mannheimia haemolytica was first reported in $1932^{[1]}$ as a cause of pneumonia, septicemia and abortion in domestic animals. ${ }^{[1]}$

P. haemolytica infection is exceedingly rare in human. Until today, it was reported only in six cases. The first human case was reported in 1962 in a case of septicemia with endocarditis, ${ }^{[6]}$ followed by a case of aortic graft infection in $1994,,^{[7]}$ a case of a respiratory infection in $1998,^{[8]}$ a case of septicemia in $2003^{[9]}$ and recently in $2012^{[1]}$ a case of pneumonia and septicemia in a 7-month-old male from rural area.

Our case is to our knowledge the first reported case of $P$. haemolytica brain abscess.

Most P. multocida brain abscesses were caused by penetrating trauma or contiguous spread from a suppurative parameningeal focus and animal exposure was frequently found. ${ }^{[4]}$ Patients with chronic pulmonary or liver disease especially when elderly seem predisposed to disseminate infection. ${ }^{[4,5]}$

Brain abscess can be less frequently hematogenously acquired. ${ }^{[4]}$

In our patient, the lack of contiguous infections and previous animal contact with the past history of cardiac IVC which is recognized as a risk factor for hematogenous brain abscess despite the absence of endocarditis and positive blood culture make the bacteremic route likely.

Regarding the 1 month evolution of the symptoms and absence of infectious syndrome, the first diagnosis suggested was erroneously a glial tumor.

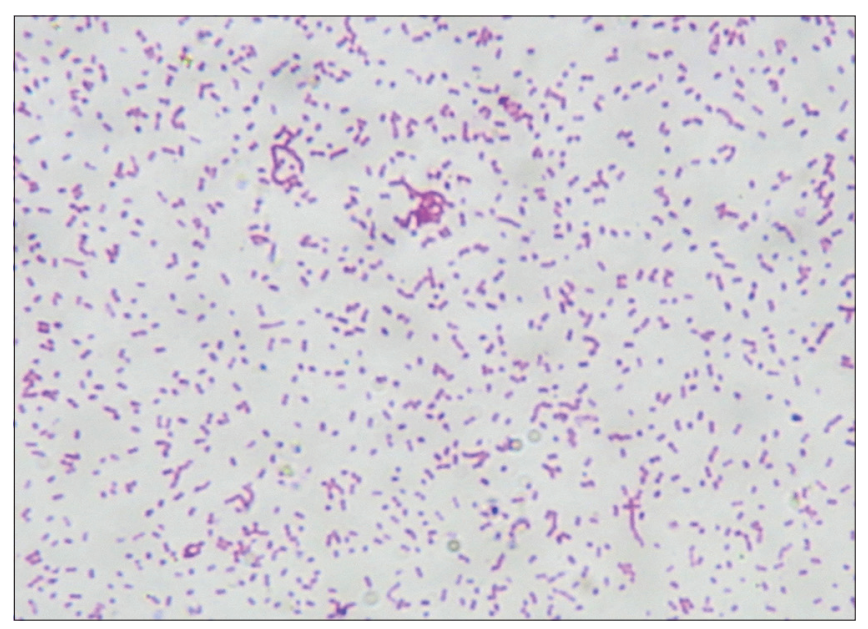

Figure 2: Gram-stained smears photomicrograph showing Pasteurella haemolytica 
The absence of infectious syndrome may be explained by the unique localized abscess with a thick wall avoiding infection spread to surrounding area.

Pasteurella abscess treatment associates aspiration or complete removal, like our patient, with antimicrobials therapy, keeping in mind the high sensitivity of Pasteurella to penicillin, ampicillin, cephalosporin and many others antimicrobials. ${ }^{[3,4]}$

Outcome depends on early diagnosis and treatment and also on initial clinical presentation such endocarditis, septicemia..., etc. ${ }^{[4,5]}$

Fatal evolution was frequently reported in Pasteurella brain abscess and explained probably by delayed diagnosis and treatment. ${ }^{[5]}$

Our patient recovered well without complications or sequellae after complete removal of her brain abscess and antimicrobials therapy.

\section{Conclusions}

Pasteurella brain abscess is exceedingly rare. It must be kept in mind in front of a suspected radiological lesion even in lack of infectious syndrome and hypertension signs and absence of animal contact.

\section{References}

1. Punpanich W, Srijuntongsiri S. Pasteurella (Mannheimia) haemolytica septicemia in an infant: A case report. J Infect Dev Ctries 2012;6:584-7.

2. Li ZX, Zhao B, Feng Z. Brain abscess due to Pasteurella multocida. Kansenshogaku Zasshi 1994;68:403-6.

3. Whittle IR, Besser M. Otogenic Pasteurella multocida brain abscess and glomus jugulare tumour. Surg Neurol 1982;17:4-8.

4. Wallace M, Lipsky BA. Hematogenous Pasteurella multocida brain abscess. West J Med 1985;143:520-3.

5. Rada N, Arrad B, Draiss G, Bourrous M, Bouskraoui M. Pasteurella multocida: A rare cause of cerebral abscess. Med Mal Infect 2012;42:525-6.

6. Doty GL, Loomus GN, Wolf PL. Pasteurella endocarditis. New Engl J Med 1963;268:830-2.

7. Rivera M, Hunter GC, Brooker J, O’Berg CW, Smythe SH, Bernhard VM. Aortic graft infection due to Pasteurella haemolytica and group C beta-hemolytic streptococcus. Clin Infect Dis 1994;19:941-3.

8. Watanabe T, Sato M, Abe T, Oda Y. Bacterial croup caused by Pasteurella haemolytica. Acta Paediatr Jpn 1998;40:360-1.

9. Takeda S, Arashima Y, Kato K, Ogawa M, Kono K, Watanabe K, et al. A case of Pasteurella haemolytica sepsis in a patient with mitral valve disease who developed a splenic abscess. Scand J Infect Dis 2003;35:764-5.

How to cite this article: Darmoul M, Nsir AB, Kilani M, Hattab MN. Hematogenous pasteurella haemolytica brain abscess. J Neurosci Rural Pract 2014;5:287-9.

Source of Support: Nil. Conflict of Interest: None declared. 